\title{
Survey on Various Techniques for Age Progression
}

\author{
Patel Mira $Y$ \\ Student \\ Department of Information \\ Technology \\ G. H. Patel College of Engineering \\ and Technology \\ Anand, India
}

\author{
Jaymit Pandya \\ Assistant Professor \\ Department of Information \\ Technology \\ G. H. Patel College of Engineering \\ and Technology \\ Anand, India
}

\author{
Nirav M. Raja \\ Assistant Professor \\ Department of Information \\ Technology \\ G. H. Patel College of Engineering \\ and Technology
}

\begin{abstract}
Age changes cause major variations in the appearance of human faces. Due to many lifestyle factors, it is difficult to precisely predict how individuals may look with advancing years or how they looked with "retreating" years. This paper is a review of age variation methods and techniques, which is useful to capture wanted fugitives, finding missing children, updating employee databases, enhance powerful visual effect in film, television, gaming field. Currently there are many different methods available for age variation. Each has their own advantages and purpose. In this paper, different age variation methods with their prospects are reviewed. These are the selected methods and techniques that had been chosen for review: Anthropometric Model, Image morphing, Image Based Surface Detail Transfer (IBSDT), aging function (AGES), Gaussian Mixture Model (GMM).
\end{abstract}

\section{Keywords}

Age progression, Age Variation Methods, Anthropometric Model, Ages, Image Morphing, Ibsdt, Gmm

\section{INTRODUCTION}

Aging is an unavoidable natural process during the lifespan of a person. Human faces are subject to change mainly due to three reasons viz. age, gender and ethnic group. As the age seems to be the main cause of the facial changes, it has come to the forefront.

Compared with other facial variations, aging effects has three unique characteristics: [2]

1. The aging progress is uncontrollable: It cannot be advanced or delayed and it is slow and irreversible.

2. Personalized aging patterns: Every people have different aging effect. The aging pattern of each person depends on his/her genes as well as many external factors, such as health, lifestyle, weather conditions, etc.

3. The aging patterns depend on time: The face status at a particular age will affect all older faces, but will not affect those younger ones.

Main Objective to build automated system for modeling the aged face at given target age is: With the passage of time the face of the person might have changed and there should be a mechanism to reveal the person's identity. In order to make this process easy, Analyst must guess and decide as to how he/she will look like by now. To address this problem this paper presents a way of effectively synthesizing a facial image with the aging effects.
There has been growing interest in face recognition which is useful in law enforcement and forensic investigation, missing individual, Multimedia. Face recognition is a wide area in research, and there are varieties of ways that it could be carried out such as Graph matching, anthropometric studies age estimation, facial feature analysis, image warping, image morphing, etc. From the previous work done in aging it can be said variety of key facial feature changes in elderly stage. Those are fine lines and wrinkles on the face, lips thin out, hair color changes, hairlines recede, balding, facial texture changes, facial shape changes, and muscles drop. However, the individual diversity is broad. Factors such as race, gender, genetics and lifestyle come into play during the aging process. [13]

Human Facial Aging can be categorized in to two main phases as formative phase and adult phase. A methodology for the formative phase (age from 0 to 18) aging is being proposed in [14]. Adult phase can be further classified as adult primary phase (age from 19 to 50) and adult secondary phase (age 50 upwards). A methodology for the adult primary phase aging is being proposed in [15]. Researcher need to focus on faces shape changes in elderly aging progression using various techniques for age variation. Most researchers used FGNET in [10] and MORPH in [9] databases for model construction and testing purposes.

\section{TECHNIQUES FOR AGE PROGRESSION}

\subsection{Anthropometric Model}

Anthropometric Model [3] is based on the craniofacial development theory and facial skin wrinkle analysis. These methods are suitable for coarse age estimation or modeling ages just for young. Since the human head shape does not change too much in its adult period. In [3] computed wrinkles from face images to separate young adults from senior adults. The wrinkles were computed in several regions, such as on the forehead, next to the eyes, and near the cheek bones. The presence of wrinkles in a region is based on the detection of curves in that region.

\subsection{Aging Pattern Subspace}

Instead of dealing with each aging face image separately, a sequence of an individual's aging face images might be used all together to model the aging process, which is called Aging pattErn Subspace (AGES)[4][5]. An aging pattern is defined as a sequence of personal face images, coming from the same person, sorted in the temporal order. If the face images of all ages are available for an individual, the corresponding aging pattern is called a complete aging pattern; otherwise, it is called an incomplete aging pattern as in Fig 1. The AGES method can synthesize the missing ages by using an EM 
(Expected Maximization)-like iterative learning algorithm. The age of a test face is determined by the projection in the subspace that can best reconstruct the face image.

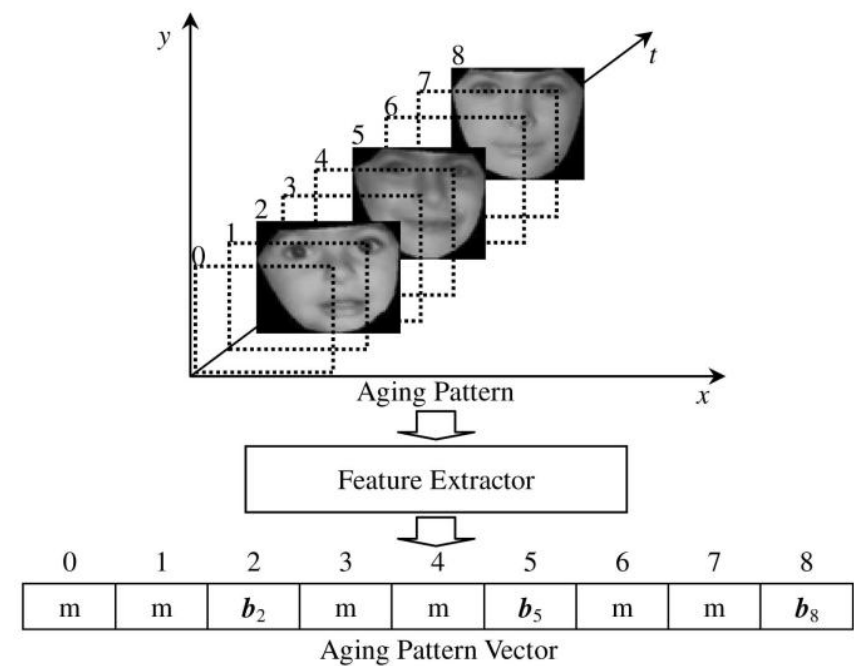

Figure 1. Vectorization of the aging pattern. The ages $(0$ 8) are marked at the top-left to the corresponding positions and above the corresponding feature vectors. The missing parts in the aging pattern vector are marked by “m." $[4][5]$

\subsection{Image Morphing}

Before the development of morphing, image transitions were commonly achieved by cross dissolves technique, which applies linear interpolation to sallow one image to another. As a result, the image looks bad because of the ghosting effects. To solve this problem, morphing is introduced. It is realized by combining image warping with cross-dissolve or color interpolation technique.

\section{Morphing Methods and Techniques:}

\subsubsection{Mesh warping}

This is an algorithm formed in two steps that accepts a source image and two 2D arrays of coordinates S and D. The S coordinates represent the control pixels in the source image, and the $\mathrm{D}$ coordinates are the locations where the $\mathrm{S}$ coordinates will match. The final image is the initial image warped by means of mesh $\mathrm{S}$ and mesh $\mathrm{D}$. The $2 \mathrm{D}$ arrays, in which the control points are stored, impose a rectangular topology to the mesh. The only constraint is that the meshes defined by both arrays be topologically. Therefore the D coordinates are coordinates that may move as far from $\mathrm{S}$ as necessary, as long as they do not intersect with themselves. [1]

The first step means re-sampling each row independently. An intermediate array of points $\mathrm{I}$, whose $\mathrm{x}$ coordinates are same as those in D and whose y coordinates are the same as those in $\mathrm{S}$, is created. Vertical splines are generated to fit each column of data in $\mathrm{S}$ and in $\mathrm{I}$. The data for each region in a row is interpolated to create intermediate image I.

The second step consists in re-sampling each column independently. Horizontal splines are then generated to fit each row of data in arrays I and D. The data for each region in a column is interpolated from intermediate image I to create destination image D.

The collection of vertical splines fitted through S and I in the first step and with the horizontal splines fitted through I and D in the second step, are shown in the figure 2.

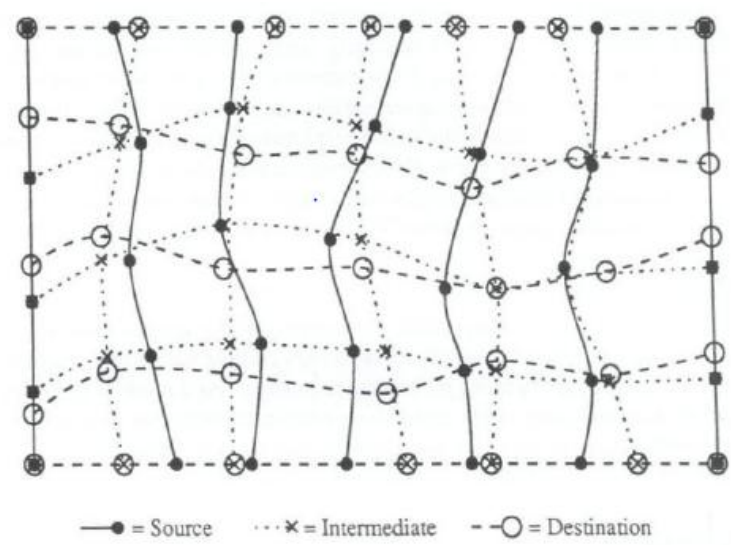

Figure 2. Mesh Warping

\subsubsection{Feature based image warping}

This is a method that offers a high level of control over the process. The corresponding feature lines in the two images that are being morphed are interactively selected. The algorithm uses lines to relate features in the source image to features in the final image. This algorithm is based upon fields of influence surrounding the feature lines selected. It uses reverse mapping for warping the image.

A pair of lines (one defined relative to the source image, the other defined relative to the destination image) defines a mapping from one image to the other (see figure 3). [1]
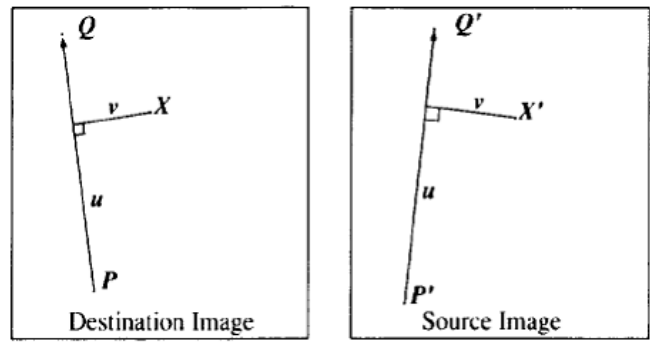

Figure 3. Feature Based Image Morphing

\subsection{Image-Based Surface Detail Transfer (IBSDT)}

Our technique-image-based surface detail transfer (IBSDT) - transfers geometric details between the images of two surfaces without knowing their 3D information [6].

One interesting application of IBSDT is aging effect synthesis. Geometrically, older people have more bumps on their facial skin surface than a young person. If researchers transfer the bumps from an old person's skin surface to a young person's face, the young person's face becomes bumpy and looks older. Conversely, they can replace an old person's skin surface bumps with a young person's skin surface so that the old person's face gets smoother and looks younger. They can apply the surface details transfer technique, on human faces to generate aging effects. They align the images by first marking face boundaries and features such as eyes, noses, and mouths, and then use triangulation-based image warping to warp I1 toward I2. They only apply IBSDT to pixels inside of the face boundary. In addition, IBSDT doesn't modify the pixels in the regions of the eyebrows, eyeballs, nose top, and mouth. [6] 
Figure 4 shows the aging effect synthesis results between the faces of a young male (Figure $4 \mathrm{a}$ ) and an old male (Figure $4 d)$

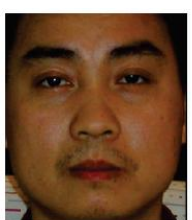

(a)

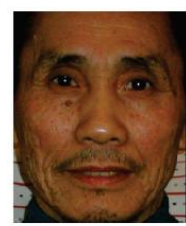

(d)

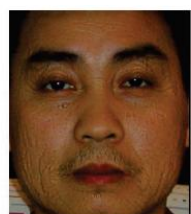

(b)

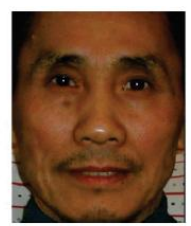

(e)

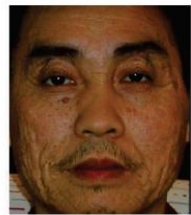

(c)

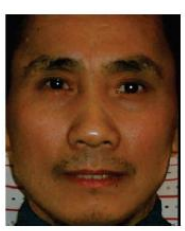

(f)
Figure 4. Young adult versus senior adult [6]

\subsection{Gaussian Mixture Model (GMM)}

It present a graph based feature representation of the face images, and build a probabilistic aging model for each individual using Gaussian Mixture Model (GMM) which incorporates both the shape and texture information. A simple graph construction algorithm is presented which uses the feature points of an image as vertices, and their corresponding feature descriptors as labels. Matching is performed in two stages. [7] In the first stage, a Maximum a posteriori solution is computed using the aging model of the individuals to effectively reduce the search space and identify potential individuals for the second stage. In the second stage, a simple deterministic graph matching algorithm that exploits the spatial similarity between the graphs is proposed.

The flow of this method as follow: [7]

1. Feature Point Extraction using Local Feature Analysis (LFA) technique.

- A modified statistical Local Feature Analysis (LFA) [16] technique is used to extract a set of feature points from each image.

2. Feature Description with Local Binary Pattern (LBP).

- A feature descriptor is constructed for each feature point extracted from an image using Local Binary Pattern (LBP). The original LBP operator proposed by Ojala et al. [17] is a simple but very efficient and powerful operator for texture description. The operator labels the pixels of an image by thresholding the $\mathrm{n} \times \mathrm{n}$ neighborhood of each pixel with the value of the center pixel, and considering the result value as a binary number. Figure 5(a) shows an example of the basic LBP operator and figure 5(b) shows two circular LBP operators with different radii.

- An extension to the original operator was also introduced by Ojala et al. [18], which uses the property called uniform patterns according to which a LBP is called uniform if there exist at most two bitwise transitions from 0 to 1 or vice versa. Uniform patterns can reduce the dimension of the LBP significantly which is advantageous for face recognition.

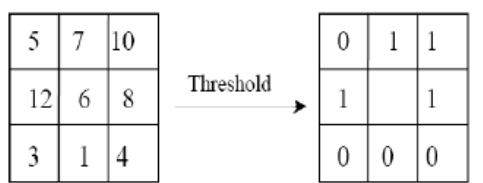

Binary: 01110001 Decimal: 113

(a) The Basic LBP operator
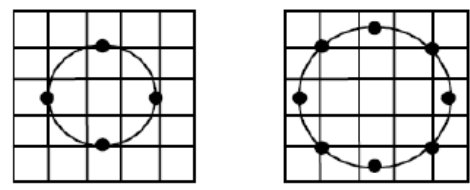

(b) $(4,1)$ and $(8,2)$ circular LBP operator

Figure 5. 5(a) The basic LBP operator. 5(b) $(4,1)$ and $(8$, 2) circular LBP operator[7].

3. Graph Construction

- Each face image is represented by a graph using the feature points as the vertices. The most distinctive property of a graph is its appearance, which is computed from the description vectors of the vertices of the graph. Graph geometry, i.e., the way the vertices of a graph are arranged spatially could vary with every graph and play an important role in discriminating the graphs of different face images. In [7], the graph geometry is defined by constructing a graph with constraints imposed on the length of the edges between a vertex and its neighbors.

4. Age Model

- Face of humans at a younger age undergoes shape changes, while face of adults undergoes textural changes than shape changes. Hence it is appropriate to include the shape and textural changes in an aging model of an individual. In [7], an age model is developed using the model graphs, extracted from the training images of an individual.

5. Graph Matching and Recognition

- In the testing stage, feature points are extracted from the probe image using the LFA technique, and the feature descriptors are computed using LBP. A graph $\mathrm{G}$ is constructed with the set of feature points obtained for the probe image. The MAP probability is computed in the first stage of matching and is used to effectively prune the search space by selecting the age model of those individuals which are likely to match with the testing image. In the second stage, [7] use a simple deterministic algorithm to match the graph $G$ with the set of graphs in the training data set.

\section{AGING DATABASES AND GROWTH RELATED DATA}

Among many available face databases around the world [8], three of them include significant sets for aging individuals.

- MORPH Database

- $\quad$ FG-NET

- FERNET Database 


\subsection{The MORPH Database [9]}

The MORPH Database comprises face images of adults taken during different ages.

The database has been organized into two albums:

- 'MORPH Album 1' and 'MORPH Album 2'.

'MORPH Album 1' comprises of 1690 digitized images of 515 individuals under the age range $15-68$ years.

'MORPH Album 2' comprises of 15204 images of nearly 4000 individuals. Apart from the face images, the database also provides meta- information that is critical for the task of studying age progression such as age, sex, ethnicity, height and weight.

\subsection{FG-NET Aging Database [10]}

The FG-NET (Face and Gesture Recognition Research Network) aging database [12] comprises of 1002 images of 82 subjects. Drifts in facial features for a few age- separated face images from the FG-Net aging database. The drifts across images of same individuals appear coherent (top two rows); while they are somewhat incoherent (third row) when the images belong to different individuals [39] (6 - 18 images per subject) in the age range $0-69$ years. The database also provides 68 landmark features that were identified manually, on all the face images. In addition, the following metainformation is available for all the images in the dataset namely: image size, age, gender, spectacles, hat, mustache, beard, horizontal pose and vertical pose. Since the images were retrieved from real-life albums of different subjects, aspects such as illumination, head pose, facial expressions etc. are uncontrolled in this dataset. Nevertheless, this database is the only publicly available resource that provides quite a few age separated face images of individuals in the age range 0 18 years.

\subsection{FERET Database [11]}

The FERET Database, that addresses multiple problems related to face recognition such as illumination variations, pose variations, facial expressions etc. also comprises of a few hundred age separated face images of subjects (the age separation amounting to 18 months or more). The FERET dataset, pertaining to facial aging, can be described as below:

Gallery-set: Comprises of 1196 images.

\section{COMPARISION}

There are a large number of algorithms and some of them have been described in this paper. To compare these algorithms, Correct Identification Rate and Mean Absolute Error (MAE) is used in most of these papers. MAE is defined as average absolute error between estimated and chronological age [20]. The lowest the MAE the more accurate the algorithm is. Most commonly used database also used in this comparison is FG-NET database and MORPH database. Algorithms by Correct Identification Rate and MAE can be seen in Table 1 .

Table 1. Comparison of different techniques

\begin{tabular}{|l|l|l|}
\hline Title, Author, & Method used & Result \\
Publication & & \\
\hline Title: Age Progression & Anthropometry & Correct \\
for Elderly People Using & Model + Image & Identification \\
Image Morphing ${ }^{[13]}$ & Morphing & Rate $=60 \%$ \\
Authors: L. L. Gayani & & \\
Kumari, and Anuja & & \\
Dharmaratne & & \\
\hline
\end{tabular}

\begin{tabular}{|c|c|c|}
\hline $\begin{array}{l}\text { Publication: IEEE, } \\
2011\end{array}$ & & \\
\hline $\begin{array}{l}\text { Title: Matching Facial } \\
\text { Images Using Aging } \\
\text { Related Morphing } \\
\text { Changes }{ }^{[15]} \\
\text { Authors: Udeni } \\
\text { Jayasinghe \& Anuja } \\
\text { Dharrnarathne } \\
\text { Publication: World } \\
\text { Academy of Science } \\
\text { Engineering \& } \\
\text { Technology, } 2009\end{array}$ & $\begin{array}{l}\text { Anthropometry } \\
\text { Model + } \\
\text { Warping } \\
\text { Algorithm }\end{array}$ & $\begin{array}{l}\text { Correct } \\
\text { Identification } \\
\text { Rate }=54 \%\end{array}$ \\
\hline $\begin{array}{l}\text { Title: Image Based } \\
\text { Surface Detail } \\
\text { Transfer }^{[6]} \\
\text { Authors: Y. Shan, Z. } \\
\text { Liu and Z. Zhang } \\
\text { Publication: Hawaii, } \\
\text { Dec } 2011\end{array}$ & IBSDT & NA \\
\hline $\begin{array}{l}\text { Title: Learning from } \\
\text { Facial Aging Patterns for } \\
\text { Automatic Age } \\
\text { Estimation }{ }^{[5]} \\
\text { Authors: X. Geng, Z.- } \\
\text { H. Zhou, Y. Zhang, G. } \\
\text { Li, and H. Dai } \\
\text { Publication: IEEE, } \\
2006\end{array}$ & AGES & $\begin{array}{l}\text { Mean } \\
\text { Absolute } \\
\text { Error }=6.77 \%\end{array}$ \\
\hline $\begin{array}{l}\text { Title: Automatic Age } \\
\text { Estimation Based on } \\
\text { Facial Aging Patterns }{ }^{[4]} \\
\text { Authors: X. Geng, Z.- } \\
\text { H. Zhou, Y. Zhang, G. } \\
\text { Li, and H. Dai } \\
\text { Publication: IEEE, } \\
2007\end{array}$ & Modified AGES & $\begin{array}{l}\text { Mean } \\
\text { Absolute } \\
\text { Error }=6.22 \%\end{array}$ \\
\hline $\begin{array}{l}\text { Title: Age Invariant } \\
\text { Face Recognition Using } \\
\text { Graph Matching }{ }^{[7]} \\
\text { Authors: Gayathri } \\
\text { Mahalingam \& Chandra } \\
\text { Kambhamettu } \\
\text { Publication: IEEE, } \\
2010\end{array}$ & $\begin{array}{l}\text { Graph and } \\
\text { Gaussian } \\
\text { Mixture Model } \\
\text { (GMM) }\end{array}$ & $\begin{array}{l}\text { Error Rate }= \\
29.2 \%\end{array}$ \\
\hline $\begin{array}{l}\text { Title: Modelling Age } \\
\text { Progression in Young } \\
\text { Faces }{ }^{[21]} \\
\text { Authors: Narayanan } \\
\text { Ramanathan, Rama } \\
\text { Chellappa } \\
\text { Publication: IEEE, } \\
\text { 2006 }\end{array}$ & $\begin{array}{l}\text { Craniofacial } \\
\text { growth model }\end{array}$ & $\begin{array}{l}\text { Correct } \\
\text { Identification } \\
\text { Rate }=58 \%\end{array}$ \\
\hline
\end{tabular}

\section{CONCLUSION}

Estimation of aging is an important aspect of applications like transformation of face from young to old for missing persons, finding intermediate faces given two faces i.e. one of young and other of old. It will hard to predict accurate face. As described in above discussion that various features of face changes under aging effect. In above survey, methods taken are answering some of changing features. These features are taken exclusively from other so that they can be modelled simplistically. However, it will give good idea of effect of changing of that feature on face, but overall prediction of face will not be exact. To have closer approximation, combination 
of these features i.e. methods should be taken and it should be matched against original image.

Human aging is an important aspect for biometrics and also for all face processing applications and not studied in depth yet. The subject has various aspects and various impacts on different disciplines. Different methods are summarised in table 1, with its respective results. Future research can be: aging is an obstacle in face recognition beside others such as beard, glasses etc. On the other hand, aging is a natural process in humans' life and there might be thousands of computer vision applications regarding this process. Age estimation is one of the major issues in those applications. And most important one is modelling the aging process. This latter may be useful in security applications such as in passport control, or finding lost children. The bottom line is: "aging" is an important aspect that has various impacts on different disciplines and a fertile ground for future research.

\section{REFERENCES}

[1] Alexandru Vlad FECIORESCU, "IMAGE MORPHING TECHNIQUES”, Volume 5 Issue 1 JIDEG, June 2010

[2] Aashmi, Sakshi Sahni, Sakshi Saxena, "SURVEY: Techniques for Aging Problems in Face Recognition", MIT International Journal of Computer Science and Information Technology, Vol. 4, No. 2, August 2014

[3] Y. H. Kwon and N. da Vitoria Lobo, "Age classification from facial images," Computer Vis. Image Understand., Vol. 74, No. 1, pp. 1-21,1999.

[4] X. Geng, Z.H. Zhou, and S.M. Kate, "Automatic age estimation based on facial aging patterns," IEEE Trans. Pattern Anal. Machine Intell, Vol. 29, No.12, pp. 2234 2240, and 2007.

[5] X. Geng, Z.H. Zhou, Y. Zhang, G. Li, and H. Dai, "Learning from facial aging patterns for automatic age estimation," in ACM Conf. Multimedia (ACM MM'06), 2006, pp. 307-316.

[6] Y. Shan, Z. Liu and Z. Zhang, "Image-Based Surface Detail Transfer," CVPR 2001, Hawaii, Vol. II, Page(s): 794-799, Dec. 2001.

[7] Gayathri Mahalingam \& Chandra Kambhamettu "Age Invariant Face Recognition Using Graph Matching" IEEE, 2010

[8] X. Geng, Z.H. Zhou, Y. Zhang, G. Li, and H. Dai, "Learning from facial aging patterns for automatic age estimation," in ACM Conf. Multimedia (ACM MM'06), 2006, pp. 307-316.

[9] K. Ricanek, T.Tesafaye, "MORPH: A Longitudinal Image Database of Normal Adult Age- progression," in
IEEE International Conferenceon Automatic Face and Gesture, 2006.

[10] FG-NET Database, http://www.fgnet.rsunit.com/

[11] M. Burt and D.I. Perrett, "Perception of age in adult Caucasian male faces: computer graphic manipulation of shape and color information," Journal of Royal Society, Vol. 259, pp. 137-143, February 1995.

[12] Y. Fu and T.S. Huang, "Human age estimation with regression on discriminative aging manifold," IEEE Trans. Multimedia, to be published.

[13] L. L. Gayani Kumari, and Anuja Dharmaratne, "Age Progression for Elderly People Using Image Morphing", The International Conference on Advances in ICT for Emerging Regions, IEEE 2011

[14] K.S. Ariyarathne \& A.T. Dharrnaratne, "Age Related Morphing Progression of Young Faces", Int'l Conf. in Machine Vision (ICMN 2010), Hong Kong, Dec. 2010

[15] Udeni Jayasinghe \& Anuja Dharrnarathne "Matching Facial Images Using Aging Related Morphing Changes" World Academy of Science, Engineering \& Technology 60, 2009

[16] P. Penev and J. Atick,'Local feature analysis: A general statistical theory for object representation," Network: Computation in Neural Systems, 7(30: 477-500, 1996.

[17] Ojala, T., Pietikainen, M., Harwood, D.,’A comparative study of texture measures with classification based on feature distributions," Pattern Recognition 51-59, 1996.

[18] Ojala, T., Pietikainen, M., Maenpaa, T.,”A generalized local binary pattern operator for multi-resolution gray scale and rotation invariant texture classification," Second International Conference on Advances in Pattern Recognition, Rio de Janeiro, Brazil (2001) 397-406.

[19] E. Patterson, A Sethuram, M. Albert, K. Ricanek, and M. King, "Aspects of age variation in facial morphology affecting biometrics," in IEEE Int Conf. on Biometrics: Theory, Applications and Systems, Crystal City, VA, 2007.

[20] G.Guo, Y.Fu, T.Huang, C.Dyer. 2008. Locally Adjusted Robust Regression for Human Age Estimation. IEEE Workshop on Application of Computer Vision.

[21] Narayana Ramanathan, Rama Chellappa, "Modeling Age Progression in Young Faces", Computer Society Conference on Computer Vision and Pattern Recognition(CVPR'06), IEEE, 2006 